\title{
SISTEMAS DE AVALIAÇÃO PRECOCE NA IDENTIFICACCÃO DE MORBIDADES MATERNAS: REVISÃO SISTEMÁTICA
}

\author{
Jarbas Galvão \\ Mestre em Desenvolvimento Regional, (FURB), SC. \\ Docente do Departamento de Enfermagem da Fun- \\ dação Universidade Regional de Blumenau (FURB), \\ Blumenau (SC), Brasil.
}

E-mail: jarbasgalvao@gmail.com

\section{Jean Carl Silva}

Doutor em Ciências Médicas (UNIFESP), São Paulo, (SP). Docente do Departamento de Medicina da Universidade Regional de Joinville e do Programa de Pós-Graduação Saúde e Meio Ambiente (UNIVILLE), Joinville, (SC), Brasil.
RESUMO: O objetivo desse trabalho foi identificar, por meio de uma revisão sistemática, os sistemas de avaliação precoce existentes que são aplicados às gestantes, descrevendo os parâmetros vitais utilizados, as semelhanças \diferenças, bem como a efetividade desses instrumentos. Trata-se de um estudo de revisão sistemática, cujas pesquisas foram realizadas utilizando as bases de dados MEDLINE, SciELO, LILACS, BIREME e PubMed sendo encontrados 191 artigos. Excluíram-se os artigos que não tinham como foco o estudo, restando 16 artigos. Os resultados encontrados foram quatro instrumentos de avaliação aplicadas em pacientes para predizer deterioração fisiológica, sendo eles: EWS, NEWS, MEWS, MEOWS. Os instrumentos apresentaram pontos positivos relevantes, como indicadores do gerenciamento do cuidado, de custo hospitalar e social, porém, recomendando-se mais estudos para refinamento. O MEOWS é a ferramenta mais apropriada às condições maternas, não validada, e que necessita de adaptação às alterações fisiológicas relacionadas às diferentes morbidades. Sugere-se aumentar o corpo de estudos sobre este instrumento a fim de melhorar a sua especificidade, tornando-a uma ferramenta mais ajustada e confiável como preditora de near miss.

PALAVRAS-CHAVE: Mortalidade materna; Morbidade; Sistema de alerta precoce; Complicações na gravidez; Near Miss.

\section{EARLY ASSESSMENT SYSTEMS IN THE IDENTIFICATION OF MATERNAL DISEASES: SYSTEMATIC REVIEW}

ABSTRACT: Early assessment systems applied to pregnant women, describing vital parameters, similarities/differences, and the efficaciousness of such tools, are analyzed. Systematic review was based on databases MEDLINE, SciELO, LILACS, BIREME and PubMed, with 191 scientific articles. The articles without the theme as focus were excluded and only 16 papers were analyzed. Results showed four evaluation tools applied to patients to predict physiological deterioration, namely, EWS, NEWS, MEWS, MEOWS. The tools had relevant positive points, such as indicators of care management, hospital and social costs, even though further studies were needed for better evaluation. MEOWS is the best tool for maternal conditions, albeit non-validated, and should be adapted to physiological changes related to different diseases. Further studies on this tool should be undertaken to improve its specificity and make it more reliable as a near miss predictor.

KEYWORDS: Maternal mortality; Morbidity; Early alert system; Complications in pregnancy; Near miss. 


\section{INTRODUÇÃO}

A intercorrência obstétrica refere-se a um evento ou acidente que requer ação imediata. Considerandose a singularidade da área obstétrica, a atenção às mulheres durante a gravidez, ao parto e ao puerpério requerem precauções dos hospitais em todos os níveis de complexidade, pelas eventuais intercorrências que agravam a saúde da mulher ou a do feto (BRASIL, 2009).

Autores como Soares (2012) e Silva (2015) atribuem à vulnerabilidade (intercorrência e/ou complicações) e às mortes materno-infantis a inadequada resposta dos serviços de saúde quanto à acessibilidade, monitoramento e a falta de organização dos sistemas de referência para o atendimento eficiente às intercorrências obstétricas. As intercorrências maternas vividas durante o ciclo gravídico-puerperal são, portanto, complicações potencialmente fatais e, dependendo da gravidade, podem resultar em internação hospitalar e são consideradas um dos mais sensíveis indicadores da falta de equidade social e pobreza, principalmente nos países em desenvolvimento (BRASIL, 2000).

De acordo com o Nakamura (2013), essas intercorrências podem ser de causa direta e compreendem as complicações obstétricas, parto e puerpério por omissões, intervenções e tratamento incorretos. Estes representam a maioria dos eventos não só no Brasil, mas no mundo, sendo na sua quase totalidade evitáveis como as complicações hipertensivas, hemorrágicas, infecciosas, metabólicas entre outras. Já as intercorrências que precedem a gravidez, ou que se agravaram pelos efeitos fisiológicos da mesma, como exemplo, as morbidades hematológicas, respiratórias, endócrinas, hipertensivas, caracterizam-se por causas indiretas.

No Brasil, as altas taxas de mortalidade materna são atribuídas às diferenças regionais, socioeconômicas e acesso aos serviços de saúde, tendo como as regióes Norte, Nordeste e Centro-oeste do país as maiores taxas de mortalidade materna relacionadas a causas diretas, sendo a hipertensão, hemorragia, aborto e infecção as principais causas de morte no Brasil (BRASIL, 2009).

Os estudos que avaliam a saúde da mulher sempre estiveram apoiados nos indicadores da mortalidade materna, por refletir a qualidade dos serviços de saúde e o atendimento oferecido à mulher durante o ciclo gravídico e puerpério. No entanto, as causas de morbidade materna assemelham-se as de mortalidade e vem recebendo importância como sendo fator preditivo para análise do óbito materno, tornando-se um indicador mais útil (BOTELHO, 2014).

Assim, as intercorrências nos seus diversos níveis passaram a ser objetos de estudos principalmente as complicações maternas graves ou que experimentaram a proximidade com a morte, que de acordo com WHO (2011), é denominado "Near Miss Materna".

Esse conceito é aplicado nos casos em que as mulheres apresentem critérios indicativos de morbidade, apresentando disfunção orgânica ou patologias mais frequentes associadas ao óbito materno e que estejam internadas em unidades especializadas de atendimento obstétrico ou em UTI (REICHENHEIM et al., 2009).

Os pacientes internados em hospitais podem apresentar subitamente deterioração em seus parâmetros vitais que estão associados a eventos adversos. No entanto, alteração de um único parâmetro nem sempre é preditor de risco, levando ao entendimento de que as morbidades estão relacionadas com alterações de dois ou mais sinais vitais. Assim quanto mais alterações maiores as chances de mortalidade (ALAM, 2014)

Estudo realizado no Reino Unido em dez hospitais no ano de 2009, por meio da revisão retrospectiva em 1.000 prontuários, teve como objetivo estimar com maior precisão o número de mortes evitáveis e descrever os problemas nos cuidados que são responsáveis por esse evento. Concluíu-se que o maior problema encontrado estava na monitoração clínica deficiente dos pacientes e erros de diagnósticos, sendo que $5,2 \%$ das mortes poderiam ter sido evitadas (HOGAN et al, 2012).

Em razão de inúmeros estudos realizados sobre mortalidade relacionada à deterioração dos padrões fisiológicos, foram desenvolvidos diferentes instrumentos de avaliação envolvendo os parâmetros vitais, de forma a auxiliar na detecção de gravidades de forma precoce.

Esses instrumentos têm por finalidade a avaliação dos parâmetros vitais e definir uma conduta a ser desenvolvida em detrimento aos achados. Eles contêm como base os quatro sinais vitais e podem ser atribuídos outros parâmetros ou ponderando os escores de acordo 
com a especificidade clínica. Nesse caso, requer que essas alterações sejam sempre validadas (SMITH, 2013).

No entanto, não se sabe quais instrumentos de avaliação precoce têm sido aplicados, de forma específica, para pacientes gestantes. Sabe-se que os sistemas de alerta precoce existentes foram desenvolvidos para avaliar a população adulta não obstétrico e a eficácia desses instrumentos, quando aplicados em gestantes, produzem resultados conflitantes (SINGH et al., 2016).

Dessa forma, o objetivo desse estudo foi identificar por meio de uma revisão sistemática, os sistemas de avaliação precoce existentes que são aplicados às gestantes, descrevendo os parâmetros vitais utilizados, as semelhanças \diferenças, bem como a efetividade desses instrumentos.

Com base nos resultados deste estudo, será possivel criar ou adaptar instrumentos que considerem as características das pacientes gestantes para aumentar o poder de assertividade e especificidade dos instrumentos.

\section{METODOLOGIA}

Para alcançar o objetivo do estudo foi realizada uma revisão sistemática dos artigos científicos publicados em revistas nas bases de dados MEDLINE, SciELO, LILACS, BIREME e PubMed. A revisão sistemática visa levantar a síntese de pesquisas publicadas pertinentes a um determinado assunto pelo uso de métodos explícitos e sistematizados de busca, avaliando a qualidade, validade e aplicabilidade desses estudos (DE-LA-TORRE-UGARTEGUANILO et al., 2011).

Para nortear a revisão formulou-se a seguinte questão: Quais sistemas de alerta precoce são utilizados para predizer as morbidades maternas?

Para iniciar a seleção de artigos para a sistematização, utilizaram-se as seguintes palavraschave: maternal morbidity, severe maternal morbidity, severe obstetric morbidity, Warning score, pregnancy complications, near miss, checkers instrument. As palavras-chave foram inseridas na busca da base de dados de forma combinada, ou seja, uma palavra-chave relacionada aos tipos de sistemas de alerta precoce e a outra, referente às morbidades maternas. Estas palavras- chave deveriam aparecer no título, resumo ou no corpo do texto de artigos científicos nacionais e internacionais. Das palavras-chave mencionadas, foram feitas 20 combinações de buscas, resultando em 191 artigos. Os artigos encontrados foram exportados para o gerenciador de referências Endnote $X 7$, e classificados quanto ao instrumento de alerta precoce do qual se referiam.

Após este procedimento foi realizada a leitura dos artigos a fim de verificar se estavam realmente relacionados com as morbidades e os instrumentos de diagnóstico precoce. Neste processo foram excluídos os artigos que possuíam as palavras-chave, mas não as tinham como foco do estudo, os relatos de caso, reflexões, dissertações e teses. Desta forma, restaram 16 artigos, sendo três destes relacionados ao instrumento EWS (Early warning score), cinco ao MEOWS (Modified Early Obstetric Warning Score), quatro ao MEWS (Modified Early Warning Score) e cinco ao NEWS (National Early Warning Score).

A estratégia de busca nesse trabalho é apresentada na Figura 1.

Os artigos selecionados foram listados (Quadro 2), qualificados quanto ao instrumento de diagnóstico precoce utilizado, autores/ano/ país, tipo de estudo e população. Por fim, foram feitas análises que descrevem os artigos e comparam os instrumentos de diagnóstico precoce, bem como os parâmetros e limiares utilizados em cada um.

\section{RESULTADOS}

Os estudos apresentados nesse artigo foram organizados por instrumento de avaliação precoce de forma a tornar mais objetiva a compreensão. Foram abordados quatro sistemas de alerta precoce obedecendo à metodologia proposta, descritos no Quadro 1. 


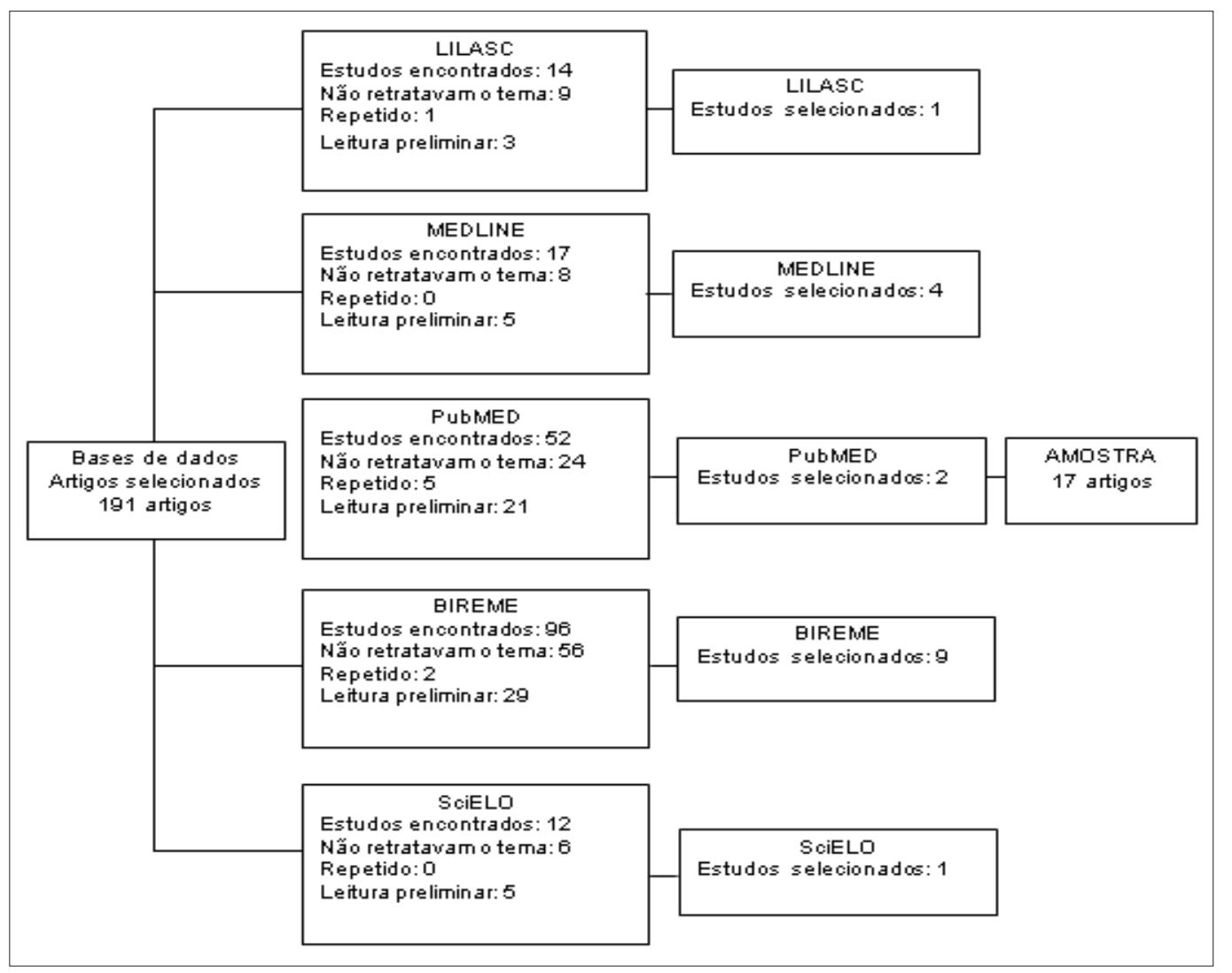

Figura 1. Fluxograma relativo à seleção dos artigos sistemas de avaliação precoce na identificação de morbidades maternas

Quadro 1. Apresentação dos instrumentos e a ordem de apresentação

\begin{tabular}{|c|c|l|}
\hline Nome & Descrição & \multicolumn{1}{c|}{ Objetivos } \\
\hline EWS & Early warning score & $\begin{array}{l}\text { Projetado para ajudar no reconhecimento e manejo apropriado de pacientes clinicamente } \\
\text { deteriorados ou em risco de deterioração clínica. É complementar a avaliação clínica } \\
\text { qualificada e tomada de decisão }\end{array}$ \\
\hline NEWS & $\begin{array}{c}\text { National Early Warning } \\
\text { Score }\end{array}$ & $\begin{array}{l}\text { Desenvolvido por UK Royal Colleges para fornecer um padrão nacional no Reino Unido } \\
\text { para Early Warning Pontuações }\end{array}$ \\
\hline MEWS & $\begin{array}{c}\text { Modified Early Warning } \\
\text { Score }\end{array}$ & Modificado para atender aos requisitos de pacientes em diferentes situações críticas. \\
\hline MEOWS & $\begin{array}{c}\text { Modified Early Obstetric } \\
\text { Warning Score }\end{array}$ & $\begin{array}{l}\text { Projetado para apoiar o uso de Track and Trigger em todas as mulheres que recebem } \\
\text { cuidados de serviços de maternidade }\end{array}$ \\
\hline
\end{tabular}

Fonte: dados da pesquisa

A seguir, conforme Quadro 2, iniciaram-se as análises dos estudos elencados em que foram identificadas apenas publicações internacionais, no idioma inglês, sendo que sete artigos (43,8\%) se referem a estudos prospectivos; cinco (31,3\%) a estudos retrospectivos; dois (12,5\%) etnográficos; um (6,2\%)multicêntrico e um (6,2\%) longitudinal.

Dentre os 16 artigos selecionados, nove (56,3\%) avaliam a aplicação dos escores de alerta precoce em pacientes graves não obstétricos; cinco (31,6\%) em pacientes obstétricas e dois $(15,5 \%)$ artigos se referem à avaliação dos escores feita por enfermeiros e médicos para verificar a adesão destes profissionais a este instrumento como ferramenta de diagnóstico de morbidades. 
Observou-se que 43,8\% das publicações foram realizadas no Reino Unido, sendo as demais publicações divididas igualmente entre África, Europa e EUA com 18,8\% cada. Os periódicos foram publicados em importantes revistas especializadas como Anaesthesia: Journal of the Association of Anaesthetists of Great Britain and
Ireland; European Journal of Gynecology Obstetrics and Reproductive Biology; Official Journal of the European Resuscitation Council; QJM: International Journal of Medicine; European Journal of Internal Medicine.

Quadro 2. Descrição dos artigos selecionados

\begin{tabular}{|c|c|c|c|c|}
\hline Instrumento & Autor & Ano/país & Tipo de estudo & População \\
\hline EWS & Subbe et al. & País de Gales/2003 & Estudo prospectivo & Clínicos e trauma \\
\hline EWS & Carle et al. & Inglaterra/2013 & Estudo retrospectivo & Pacientes gestantes \\
\hline EWS & Correia et al. & Portugal/2014 & Estudo retrospectivo & Clínicos e trauma \\
\hline NEWS & Eccles et al. & Reino Unido/2014 & Estudo prospectivo & Clínico (DBPOC) \\
\hline NEWS & Alam et al. & Holanda/2015 & Estudo prospectivo & Clínicos e trauma \\
\hline NEWS & Abbott et al. & Reino Unido/2016 & Estudo prospectivo & Pacientes clínicos \\
\hline NEWS & Hoikka et al. & Finlândia/2016 & Estudo retrospectivo & Clínicos e trauma \\
\hline MEWS & Lappen et al. & Chicago/2010 & Estudo retrospectivo & Pacientes obstétricas \\
\hline MEWS & Cooksley et al. & Reino Unido/2012 & Estudo retrospectivo & Pacientes oncológicos \\
\hline MEWS & Recine et al. & Italia/2015 & Estudo multicêntrico & Clínicos e traumas \\
\hline MEWS & Kruisselbrink et al. & Uganda/2016 & Estudo prospectivo & Clínicos e traumas \\
\hline MEOWS & Singh et al. & Newcastle/2012 & Estudo prospectivo & Pacientes obstétricas \\
\hline MEOWS & Mackintosh et al. & Reino Unido/2014 & Estudo etnográfico & $\begin{array}{l}\text { Enfermeiras e } \\
\text { médicos }\end{array}$ \\
\hline MEOWS & Isaacs et al. & Reino Unido/2014 & Estudo etnográfico & Médicos anestesistas \\
\hline MEOWS & Singh et al. & India/Delhi/2016 & Estudo prospectivo & Pacientes obstétricas \\
\hline MEOWS & Merriel et al. & Zimbábue/2017 & Estudo longitudinal & Pacientes obstétricas \\
\hline
\end{tabular}

Fonte: Dados da pesquisa

Nos estudos levantados, os sistemas de alerta precoce são compostos pelos quatro sinais vitais (P,R,T,PA) além de cada instrumento apresentar outros parâmetros fisiológicos específicos, tais como $\mathrm{Sp}_{2}$, Avaliação neurológica, PAS e débito urinário.

Dos 16 artigos alçados, três deles descrevem a aplicação da ferramenta EWS (Early Warning Scores) em pacientes adultos internados em hospital, com o propósito de avaliar a aplicação desse instrumento na taxa de internação em UTI, unidades de cuidados intermediários, serviço de emergência, parada cardiorrespiratória e morte. Sendo que um dos estudos avaliou o modelo de pontuação do EWS em pacientes obstétricas com 604.846 admissões (CARLE et al., 2013).
Os resultados nos três artigos demonstraram que o instrumento EWS (Early Warning Scores) revelou não ter significância preditiva de morbidades na admissão dos pacientes ou de internação em UTI, apenas uma tendência mais precoce do encaminhamento para essa unidade e uma relação maior entre os pacientes mais velhos pela deterioração aguda dos mesmos (SUBBE et al., 2003; CORREIA et al., 2014). Quando aplicado em pacientes obstétricas, não conseguiu categorizar as pacientes de acordo com a gravidade das doenças (CARLE et al., 2013).

Outros quatro artigos descrevem o emprego do instrumento de avaliação denominado NEWS (National Early Warning Score), sendo uma ferramenta para avaliar o risco de gravidade em pacientes com mal-estar agudo 
no ambiente hospitalar (ISAACS, 2014).

Os estudos que abordam essa ferramenta foram desenvolvidos apenas em paciente adultos não obstétricos, sendo que dos quatro artigos, três fazem comparação com outros sistemas de avaliação específicos e um avaliou 274 pacientes graves durante a internação hospitalar, sendo considerado um modelo eficiente no monitoramento durante a internação para avaliar sinais de deterioração e intervir antecipadamente, mas não como um sistema de triagem (ALAM et al, 2015)

Entre os artigos de comparação, o NEWS foi analisado com o sistema de alerta CREWS (Cronic Respiratory Early Warning Score), empregado em pacientes com patologias respiratórias crônicas e o sistema EMS (Emergency Medical Services), empregado no atendimento pré-hospitalar, que categoriza os atendimentos por prioridades de gravidade. Nesses dois estudos, o objetivo foi melhorar a especificidade dos escores do NEWS e possibilitar o seu emprego nesses casos (ECCLES et al., 2014; HOIKKA et al, 2016;).

O estudo concluiu que o NEWS é afetado por doenças crônicas e pode superestimar o verdadeiro risco médico entre os pacientes. Como sendo um sistema de pontuação fisiológico, não poderá fornecer ao profissional da regulação as informações obtidas na cena, sendo melhor empregado no ambiente hospitalar (HOIKKA et al., 2016).

Já com os biomarcadores sanguíneos (lactose, glucose ou excesso de base), o objetivo foi avaliar a associação desses com sistema de pontuação NEWS, por serem sensíveis e sofrerem alterações frequentes em doenças agudas, sendo considerados variáveis contínuas (ABBOTT et al., 2016). Como desfecho, não houve valor preditivo do NEWS com os biomarcadores escolhidos ou a sua associação com o tempo de internação hospitalar (ABBOTT et al., 2016).

O terceiro instrumento de avaliação utilizado em pacientes graves encontrado na literatura foi $o$ MEWS (Modified Early Warning Score). Os resultados destacaram quatro artigos relacionados ao objetivo de busca proposto, sendo três avaliando pacientes clínicos e um paciente obstétrico.

A partir da análise desses estudos, identificouse que essa ferramenta, quando empregada para avaliar pacientes internados em enfermarias e pacientes oncológicos predizendo possíveis morbidades, não tem sido mostrado como o melhor preditor. As apreciações dos resultados apontam que essas ferramentas contêm pobre valor discriminatório preditivo, ou seja, faz-se necessário incluir parâmetros de análise fisiológicos mais específicos e uma reavaliação nas pontuações dos parâmetros existentes (COOKSLEY et al., 2012; RECINE et al., 2015; KRUISSELBRINK et al., 2016).

Com relação ao estudo que compara os critérios do MEWS e SIRS- (Systemic Inflammatory Response Syndrome) em mulheres grávidas com corioamnionite em uma população de 15.027 gestantes, conclui-se que os critérios de SIRS e MEWS não foram instrumentos precisos na identificação de pacientes em riscos de transferência para UTI, sepse ou morte entre mulheres grávidas com morbidade febril, por apresentarem elevadas taxas de valores falsos positivos (LAPPEN et al., 2010).

Algumas considerações foram levantadas em detrimento ao resultado: há diferença nas taxas metabólicas da gravidez em comparação com mulheres adultas não grávidas, e todos os pacientes que satisfazem os critérios SIRS preencheriam os critérios para sepse, porque todos os pacientes tinham clinicamente documentado infecção. Sugerem-se estudos adicionais para desenvolver modelos preditivos apropriados para mulheres grávidas (LAPPEN et al., 2010).

O quarto instrumento de avaliação encontrado na literatura consultada chama-se MEOWS (Modified Early Obstetric Alert System). Esse instrumento foi construído para avaliar somente pacientes obstétricas internadas, por meio da avaliação e correlação dos parâmetros fisiológicos com a morbidade materna (NIRMAL, 2016). Foram classificados cinco artigos para esse instrumento.

Por meio da análise dos estudos, observou-se que três artigos foram empregados para avaliar pacientes e dois referem-se à avaliação do MEOWS por profissionais enfermeiros e médicos, com o objetivo de medir a adesão dessa ferramenta como instrumento de diagnóstico de morbidades em seus processos de trabalho.

Em relação aos artigos que avaliaram a aplicação do instrumento MEOWS como uma ferramenta para prever a morbidade materna, os estudos levantados foram unânimes em acordar que se trata de uma ferramenta útil 
na predição de morbidade materna, tanto o seu uso em sala de parto quanto em mulheres submetidas à cesariana (SINGH et al.,2012; 2016; MERRIEL et al., 2017).

Referente aos estudos que avaliam o MEOWS como uma ferramenta útil de avaliação na deterioração fisiológica, os resultados apontaram como sendo um bom instrumento de comunicação entre os profissionais enfermeiros e médicos conforme pesquisa realizada em duas maternidades de referência no Reino Unido (MACKINTOSH et al., 2014). Como também, em outra pesquisa realizada no mesmo país com 205 profissionais anestesistas obstétricos, o MEOWS se constitui em um instrumento confiável e utilizado por $45 \%$ desses profissionais, sendo $91 \%$ dos respondentes concordam com os parâmetros fisiológicos descritos, afirmando ser um instrumento útil e confiável (ISAACS et al., 2014).

\section{DISCUSSÃO}

A morbimortalidade materna configura-se como um problema de saúde pública mundial, constituindo-se no Brasil um importante indicador de desenvolvimento humano e da iniquidade social, reflexo da baixa qualidade da atenção prestada ao sistema de saúde materna (NAKAMURA, 2013)

Semelhante a esta situação, países como Iraque, Somália, Etiópia e Eritreia apontam a baixa qualidade dos atendimentos prestados às gestantes pelo sistema de saúde regional, elevando o índice de mortalidade materna (JABIR et al., 2013).

No entanto, essa questão não se restringe apenas em países menos desenvolvidos. A mortalidade materna atinge também um número cada vez maior de mulheres nos países desenvolvidos por causas indiretas, decorrentes de complicações de doenças pré-existentes à gravidez (MATIAS et al., 2009).

De acordo com a Organização Mundial da Saúde (OMS), 20 milhões de mulheres anualmente apresentam transtornos graves durante a gestação e aproximadamente 500 mil mulheres vão a óbito durante o ciclo gravídico (WHO, 2011).

Diante dessa problemática em desenvolver estratégias para melhoria da saúde materna, há um crescente interesse da comunidade científica internacional para reduzir a mortalidade, focando na morbidade materna grave ou near miss como novo indicador para auxiliar na compreensão dos problemas da saúde da população obstétrica (PATTINSON, 2003).

Assim, a disfunção orgânica ou complicações maternas foram melhor estudadas por meio da medição dos parâmetros vitais e o desenvolvimento de sistema de alerta precoce com o objetivo de monitorar as mulheres durante a gravidez e puerpério.

As ferramentas de alerta precoce são sistemas simples que têm por função medir o risco de deterioração fisiológica do paciente, por meio da medição dos parâmetros fisiológicos e detectar possíveis alterações encontradas, permitindo atuar diretamente sobre a causa (WHEELER et al., 2013).

Grande número de sistemas de alerta precoce foi desenvolvido na população não obstétrica e implementados por todo o mundo, na tentativa de antever deterioração e reduzindo a ocorrência de eventos adversos em pacientes internados em UTI, e serviços de emergências (KYRIACOS et al., 2014).

A maioria desses instrumentos é construído por enfermeiros ou médicos por meio das experiências clínicas e aplicados no ambiente laboral. Isto justifica, por exemplo, a existência de inúmeras ferramentas desenvolvidas e utilizadas na mesma área, em hospitais diferentes (ALLAN et al.,2014). No entanto, para que esta ferramenta de avaliação seja de valor, ela deve ser segura, simples e validada pela sua sensibilidade, especificidade e precisão global (GUIMARÃES et al., 2015).

Como as internações, em UTI e morte, são raras na população obstétrica em países desenvolvidos, raros são os estudos que abordam uma ferramenta para avaliar essa população. No presente estudo, observa-se a presença de apenas um instrumento de alerta precoce, denominado MEOWS, adaptado à população obstétrica, que correlaciona os parâmetros fisiológicos com a morbidade materna, (NIRMAL, 2016).

Essa ferramenta foi desenvolvida no Reino Unido em 2007, a partir da simples observação dos escores fisiológicos alterados em uma tabela de observação de cabeceira, como precedentes a um evento crítico. Após o levantamento dos pacientes susceptíveis a terem morbidade, uma avaliação mais acurada é realizada para confirmação do evento (SINGH et al.,2016). 
Esse instrumento é de fácil utilização e compreende os seguintes parâmetros: $\mathrm{Fc}, \mathrm{Fr}, \mathrm{T}^{\mathrm{o}}$, PA, nível de consciência AVDI e débito urinário com variação de escores de -3 a +3 . Após a avaliação do paciente, utilizando essa escala, segue um padrão de reavaliação de acordo com a pontuação do escore apresentado ou segue as medidas padronizadas específicas desse documento (NIRMAL, 2016).

Ressalta-se que não é um instrumento definidor de tratamento ou substitui o exame físico, mas uma importante ferramenta para auxiliar no reconhecimento precoce de mulheres em deterioração (SINGH et al.,2016).

De acordo com os artigos levantados nesse estudo sobre o MEOWS, a ausência de registros dos parâmetros vitais nos instrumentos de avaliação utilizados, impactou nos resultados de muitas pesquisas para a sua validação como um modelo de escore que possa ser fidedigno e utilizado universalmente, deixando-as vulneráveis em termos de robustez e precisão (MACKINTOSH et al., 2014)

Assim, vários estudos estão sendo realizados no Reino Unido a fim de ajustar o valor dos escores. $O$ instrumento tem alta sensibilidade, especificidade razoável e precisão que reflete baixa prevalência de morbidade na população materna daquele país (SINGH et al.,2016).

Apesar das limitações é de consenso entre os pesquisadores apoiar fortemente o uso da ferramenta MEOWS como forma de torná-la uma ferramenta com maior precisão por meio da implementação da escala em pacientes obstétricos, tendo a educação continuada como uma estratégia importante para a adesão dos profissionais com os sistemas de alerta precoce (ISAACS, et al., 2014).

\section{CONCLUSÃO}

O estudo possibilitou uma síntese sobre a mortalidade materna e o conceito de near miss como foco no reconhecimento da deterioração fisiológica pelo uso de sistemas de alerta precoce.

No entanto, as ferramentas existentes para avaliar pacientes adultos não obstétricos, não são indicados para predizer as morbidades maternas, pois pelas mudanças fisiológicas na gravidez, torna a pontuação desses instrumentos inadequada e nenhuma ferramenta para avaliar as pacientes obstétricas atualmente foi validada.

Em literatura consultada, verifica-se a existência de um único instrumento, o MEOWS, como ferramenta mais apropriada às condições maternas. No entanto, necessita de adaptação às alterações fisiológicas relacionadas às diferentes morbidades obstétricas.

Como desfecho, observa-se a inexistência de uma ferramenta validada como preditoras do near miss materno. Desta forma, como pesquisa futura, sugere-se aumentar o corpo de estudos sobre esta ferramenta a fim de melhorar a sua especificidade, tornando-se uma ferramenta mais ajustada e confiável como modelo único na avaliação das pacientes obstétricas.

\section{REFERÊNCIAS}

ABBOTT, T. E. et al. A single-centre cohort study of National Early Warning Score (NEWS) and near patient testing in acute medical admissions. Eur J Intern Med, v. 35, p.78-82, nov. 2016. Disponível em: <http://www. ncbi.nlm.nih.gov/pubmed/27346295>.

ALAM N, et al. The impact of the use of the Early Warning Score (EWS) on patient outcomes: A systematic review. Resuscitation, v. 85, n. 5,p. 587-594, 2014.

ALAM, N. et al. Exploring the performance of the National Early Warning Score (NEWS) in a European emergency department. Resuscitation, v. 90, p. 111-115, 2015.

BOTELHO, N. M. et al. Causes of maternal death in Pará State, Brazil. Revista Brasileira de Ginecologia e Obstetrícia, v. 36, n. 7, p. 290-295, 2014.

BRASIL. Ministério da Saúde. Secretarias de Políticas de Saúde. Área Técnica da Saúde da Mulher. Urgências e emergências maternas: guia para diagnóstico e conduta em situações de risco de morte materna. Brasília (DF): Ministério da Saúde, 2000. Disponível em: <http:// bvsms.saude.gov.br/bvs/publicacoes/0105urgencias. $\operatorname{pdf}>$.

BRASIL. Ministério da Saúde. Secretaria de atenção à 
saúde. Departamento de ações programáticas estratégicas. Gestação de alto risco. 5. ed. Brasília: 304, 2009. (Serie A. Normas e manuais técnicos).

CARLE, C.; ALEXANDER, P.; COLUMB, M.; JOHAL, J. Design and internal validation of an obstetric early warning score: secondary analysis of the Intensive Care National Audit and Research Centre Case Mix Programme database. Anaesthesia, v. 68, n. 4, p. 354-367, Apr 2013. Disponível em: <http://www.ncbi.nlm.nih.gov/ pubmed/23488833>.

COOKSLEY, T. KITLOWSKI, E.; HAJI-MICHAEL, P. Effectiveness of Modified Early Warning Score in predicting outcomes in oncology patients. Qjm: An International Journal of Medicine, v. 105, n. 11, p. 1083-1088, 2012.

CORREIA, N. et al. Improving recognition of patients at risk in a Portuguese general hospital: results from a preliminary study on the early warning score. International Journal of Emergency Medicine, Berlin/ Heidelberg, v. 7, n. 1, p. 1-8, 2014.

DE-LA-TORRE-UGARTE-GUANILO, M. C. at al. Revisão sistemática: noções gerais. Revista da Escola de Enfermagem USP, São Paulo, v. 45, n. 5, p. 1260-1266, out. 2011. Disponível em: < http://www.scielo.br/scielo. php?>. doi: http://dx.doi.org/10.1590/

ECCLES, S. R. et al. Improving specificity whilst maintaining sensitivity of the National Early Warning Score in patients with chronic hypoxaemia. Resuscitation, v. 85, n. 1, p. 109-111, 2014.

GUIMARÃES, P. V et al. Validação de instrumento para avaliação de pacientes graves em ventilação mecânica, segundo o ABCDE. Revista Eletrônica de Enfermagem, v. 17, n. 1, p. 43-50, 2015. I

HOGAN, H. et al. Preventable deaths due to problems in care in English acute hospitals: a retrospective case record review study. BMJ quality \& safety, 2012.

HOIKKA,M.; LÄNKIMÄKI,S.; SILFVAST,T.; ALA-KOKKO, T.I. Medical priority dispatch codes-comparison with National Early Warning Score.(Report). Scandinavian Journal of Trauma, Resuscitation and Emergency
Medicine, v. 24, n.1, 2016.

ISAACS, R. A. et al. A national survey of obstetric early warning systems in the United Kingdom: five years on. Anaesthesia, v. 69, n. 7, p. 687-692, 2014.

JABIR, M. et al. Maternal near miss and quality of maternal health care in Baghdad, Iraq. BMC Pregnancy and Childbirth, v. 13, n. 1, p. 11, 2013.

KYRIACOS, U. et al. Monitoring vital signs: development of a modified early warning scoring (MEWS) system for general wards in a developing country. PLoS One, v. 9, n. 1, p. e87073, 2014.

KRUISSELBRINK, R. et al. Modified Early Warning Score (MEWS) Identifies Critical Illness among Ward Patients in a Resource Restricted Setting in Kampala, Uganda: A Prospective Observational Study. PLoS One, San Francisco, v. 11, n. 3, 2016.

LAPPEN, J. R.; KEENE, M.; LORE, M.; GROBMAN, W. A.; GOSSETT, D. R. Existing models fail to predict sepsis in an obstetric population with intrauterine infection. American Journal of Obstetrics and Gynecology, v. 203, n. 6, p. 573, 2010.

MACKINTOSH, N. et al. Recent Findings from King's College Advance Knowledge in Health and Medicine [Value of a modified early obstetric warning system (MEOWS) in managing maternal complications in the peripartum period: an ethnographic study].(Report). Health \& Medicine Week, p. 3197, 2014.

MATIAS, J.P. et al. Comparison of two methods for the investigation of maternal mortality in a municipality of the Brazilian Southeast. Rev Bras Ginecol Obstet. v. 31, n. 11, p. 559-565, 2009.

MERRIEL, A. et al. Implementation of a modified obstetric early warning system to improve the quality of obstetric care in Zimbabwe. International Journal of Gynecolog y \& Obstetrics, v. 136, n. 2, p. 175-179, 2017.

NAKAMURA,P.M. et al. Sistema de Informações Hospitalares do Sistema Único de Saúde (SIH-SUS): uma avaliação do seu desempenho para a identificação do near miss materno. Cad Saude Publica, v. 29, n. 7, p. 
1333-1345, 2013.

NIRMAL, GHATA M.; RAMACHANDRAN, ARUN. Dispersion of a passive tracer in the pressure-driven flow of a noncolloidal suspension Soft Matter, v. 12, n. 38, p. 79207936. 2016.

PATTINSON, R. C. Near misses: a useful adjunct to maternal death enquiries. Br Med Bull, v. 67, p. 231-43, 2003.

RECINE,U. et al. The change of hospital internal medicine: A study on patients admitted in internal medicine wards of 8 hospitals of the Lazio area, Italy. Italian Journal of Medicine, v. 9, n. 3, p. 252-259, 2015.

REICHENHEIM, M. E. et al. G. Severe acute obstetric morbidity (near-miss): a review of the relative use of its diagnostic indicators. Archives of gynecology and obstetrics, v. 280, n. 3, p. 337-343, 2009.

SILVA, L. A. et al. A qualidade de uma rede integrada: acessibilidade e cobertura no pré-natal. Rev. pesqui. cuid. fundam.(Online), v. 7, n. 2, p. 2298-2309, 2015.

SINGH,S. et al. A validation study of the CEMACH recommended modified early obstetric warning system (MEOWS). Anaesthesia, Oxford, UK, v. 67, n. 1, p. 12-18, 2012. .

SINGH,A. et al. Evaluation of maternal early obstetric warning system (MEOWS chart) as a predictor of obstetric morbidity: a prospective observational study. European Journal of Obstetrics and Gynecology, v. 207, p. 1117, 2016.

SMITH, G. B. Have we found the perfect early warning score? A view of ViEWS. Resuscitation, v. 84, n. 6, p. 707 708, 2013.

SOARES,V.M.N. et al. Causas de mortalidade materna segundo níveis de complexidade hospitalar. Rev Bras Ginecol Obstet, p. 536-543, 2012.

SUBBE, C. P. et al. Validation of a modified Early Warning Score in medical admissions. QJM, v. 94, n. 10, p. 521526, 2003.

SUBBE, C. P. et al. Effect of introducing the Modified Early Warning score on clinical outcomes, cardio? pulmonary arrests and intensive care utilisation in acute medical admissions*. Anaesthesia, Oxford, UK, v. 58, n. 8, p. 797-802, 2003.

WHEELER, I, et al. Early warning scores generated in developed healthcare settings are not sufficient at predicting early mortality in Blantyre, Malawi: a prospective cohort study. PLoS One, v. 8,n. 3, p. e59830, 2013.

WORLD HEALTH ORGANIZATION. Evaluating the quality care for severe pregnancy complications: The WHO near miss approach for maternal health. WHO, Geneva, 2011.

Recebido em: 2017-06-01 Aceito em: 2017-11-23 